\title{
Image dialectique et contamination dans La ricotta de Pier Paolo Pasolini
}

Xavier Vert

\section{(2) OpenEdition}

12 Journals

Édition électronique

URL : http://journals.openedition.org/imagesrevues/284

DOI : 10.4000/imagesrevues.284

ISSN : 1778-3801

Éditeur :

Centre d'Histoire et Théorie des Arts, Groupe d'Anthropologie Historique de l'Occident Médiéval, Laboratoire d'Anthropologie Sociale, UMR 8210 Anthropologie et Histoire des Mondes Antiques

\section{Référence électronique}

Xavier Vert, « Image dialectique et contamination dans La ricotta de Pier Paolo Pasolini », Images Revues [En ligne], Hors-série 2 | 2010, mis en ligne le 01 janvier 2010, consulté le 03 février 2021. URL : http://journals.openedition.org/imagesrevues/284 ; DOI : https://doi.org/10.4000/imagesrevues.284

Ce document a été généré automatiquement le 3 février 2021.

Images Re-vues est mise à disposition selon les termes de la Licence Creative Commons Attribution Pas d'Utilisation Commerciale 4.0 International. 


\title{
Image dialectique et contamination dans La ricotta de Pier Paolo Pasolini
}

\author{
Xavier Vert
}

1 La ricotta est le film des vicissitudes du tournage d'un film : un réalisateur (Orson Welles) met en scène la Passion du Christ sous forme de tableaux vivants empruntés à la peinture dite maniériste ou anticlassique italienne. Se dégage un personnage, Stracci, figurant le bon larron crucifié à côté du Christ, dont les préoccupations contrastent avec celles des autres acteurs mais dont le destin se superpose à celui de l'histoire racontée par le film dans le film: la Passion et la mort.

2 On peut également résumer La ricotta de la façon suivante: Stracci («haillons» ou encore «lumpen») est un personnage doublement exclu de l'histoire, capitaliste et marxiste, bourgeoise et ouvrière. Sans calcul possible sur le passé et le futur, il est assigné au présent absolu du besoin.

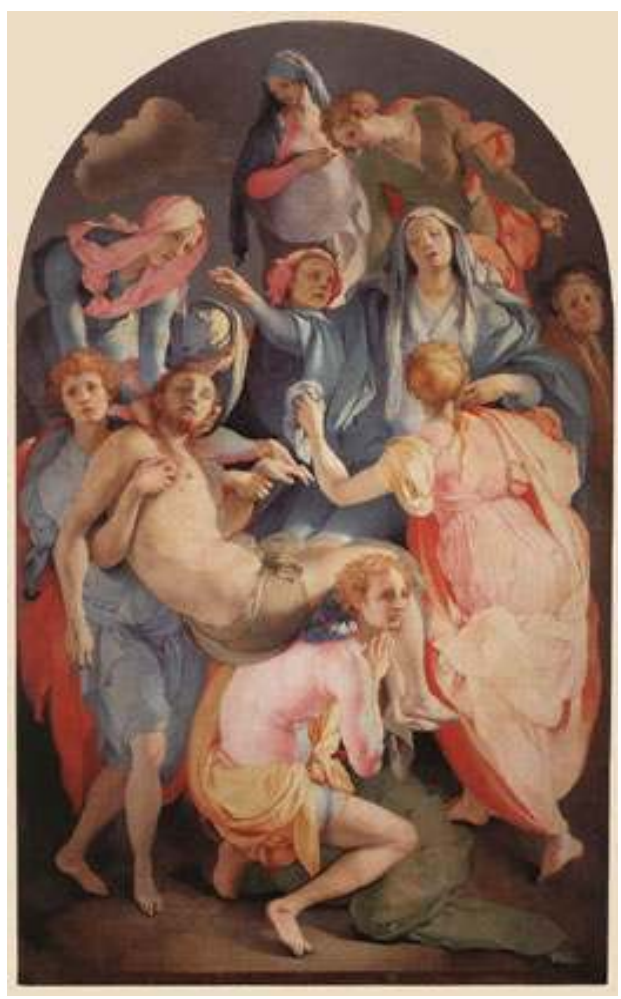
Dépouillé de toute forme politique, il est le représentant de la vie naturelle et physiologique. Ce statut d'exception, qui l'amène à endosser le rôle de figurant dans le tournage d'un film sur la Passion du Christ, fait de lui un pauvre Christ (il est le seul à pouvoir actualiser le sublime chrétien) et un bouffon qui se prendrait pour le Christ (sa 
passion et sa mort sont un renversement parodique de la Passion et de la mort du Christ). La ricotta met en scène le devenir contre figure d'un figurant de l'histoire.

3 La reconstitution vivante et la transcription cinématographique du retable peint par Pontormo pour la chapelle Capponi à Santa Felicita à Florence (fig. 1) et de la Déposition de Volterra peinte par Rosso Fiorentino en 1521 (fig. 2) constituent deux moments clé dans ce processus et de superposition et de devenir ${ }^{1}$.

Fig. 1
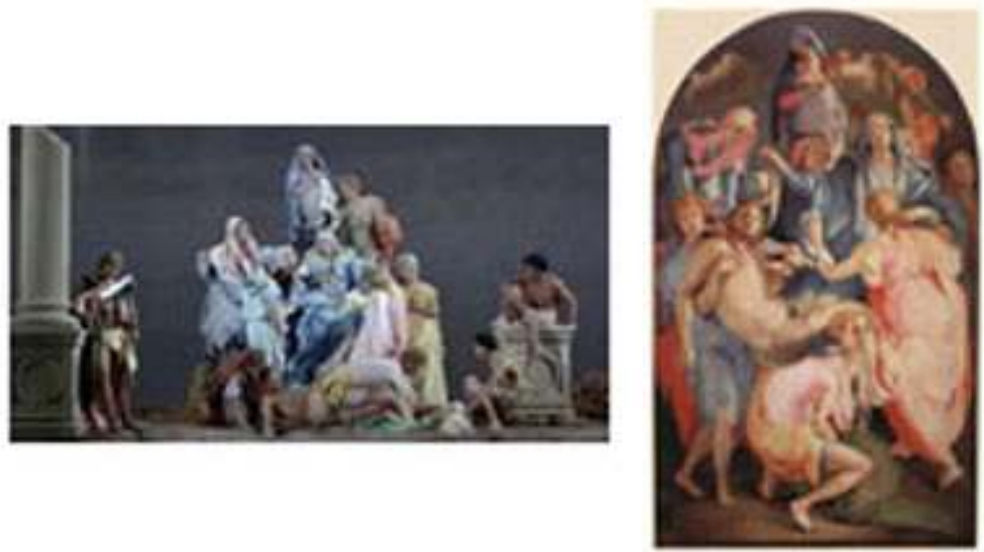

Pontormo, Déposition, (1527), huile sur bois (313 x 192), Chapelle Capponi, Eglise de Santa Felicita, Florence; Pier Paolo Pasolini, La ricotta (photogramme).

Fig. 2
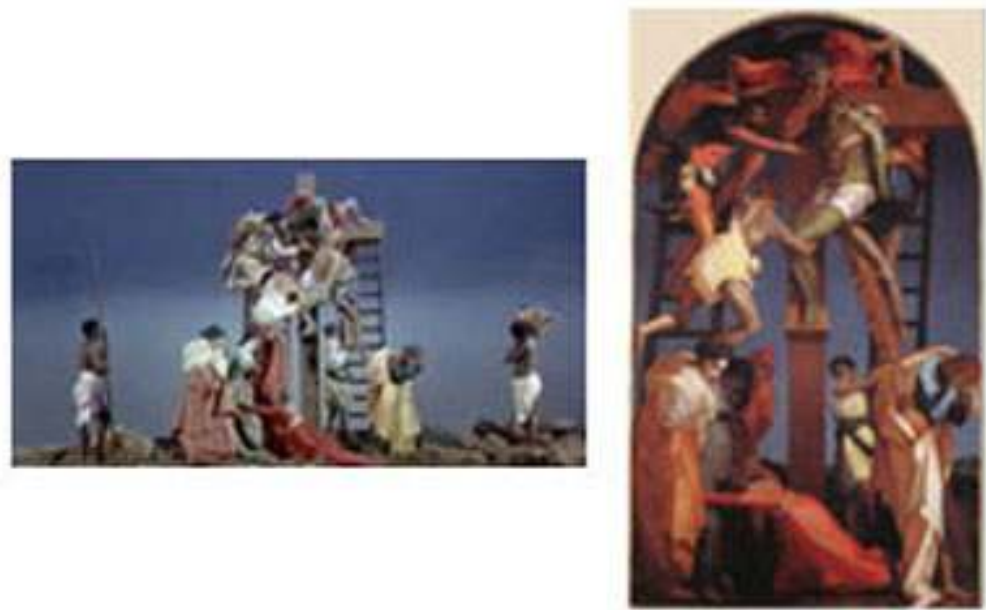

Rosso Fiorentino, Déposition de croix, (1521), huile sur bois, $(375 \mathrm{~cm} \times 196 \mathrm{~cm})$ Pinacothèque de Volterra ; Pier Paolo Pasolini, La ricotta (photogramme).

\section{L'intégration figurale du passé}

Dans un bref texte intitulé Le sentiment de l'histoire ${ }^{2}$, Pasolini concède à son interlocuteur, Carlo Lizzani, que "par nature le cinéma ne peut représenter le passé, [il] représente la réalité à travers la réalité, un homme à travers un homme ». Dans le cas d'un film historique d'auteur, «le passé lui-même devient une métaphore du 
présent », mais, ajoute Pasolini, « au sein d'un rapport complexe, parce que le présent est l'intégration figurale du passé $»^{3}$. S'agissant de La ricotta, sketch qui n'est pas un film historique, mais bien un film dont l'argument théorique est tiré des rapports anachroniques entre le passé figural et le présent de la représentation cinématographique, la question se reporte sur les conditions, les modalités et la dynamique de cette intégration figurale du passé quand elle est intégration du passé figural.

5 Le problème ainsi posé devient celui de savoir comment l'on fait revivre les images du passé4.

6 Ce problème n'est évidemment pas inédit, c'est même l'un des problèmes fondamentaux de la culture de l'image dans la tradition occidentale : Aby Warburg lui a donné le nom de "survivance » et Walter Benjamin celui de "réfraction». Or, cette grande question dont il hérite, Pasolini la met en œuvre dans La ricotta sous une forme particulière qu'il nomme "contamination». Je dirais alors que La ricotta expose spécifiquement le lien anthropologique avant qu'esthétique qui unit le thème de la contamination en tant que tel à l'histoire qui le met en scène et en image : la passion et la mort du Christ.

7 On doit à Pietro Montani la lecture la plus féconde de La ricotta; elle s'appuie sur l'analyse warburgienne de l'image en terme de schématisme, de Nachleben («vie posthume ») et d'intensification pathétique: "Dans l'art figuratif, la survivance du passé est une constante. Plus encore, [...] cette "vie posthume" est un des phénomènes les plus insistants et inquiétants de la figuration. Les images ont le pouvoir de revenir, de faire revivre leur pathos dans d'autres images et sous des formes différentes. Mais qu'est-ce que ce retour? Que transporte ce caractère posthume? De quelle façon ce passé et cette mémoire interviennent-ils dans la dramaturgie de la forme cinématographique? $»^{5}$ La thèse de Montani est la suivante: le phénomène de Nachleben joue dans La ricotta un rôle prépondérant, car ce n'est pas seulement à travers la citation que le cinéma témoigne de la persistance en lui de son propre passé figural ou que l'image cinématographique énonce une dette à l'égard de la peinture; plus fondamentalement, Pasolini expose et met en scène le processus même du retour et le "schématisme » de l'image, comme le dit Warburg. Autrement dit, dans La ricotta la "dramaturgie de la forme cinématographique» est dramaturgie même de la "survivance" et la dramaturgie de la survivance se fait dramaturgie de la forme cinématographique.

8 De son côté, Walter Benjamin affirmait qu'il importe autant de circonscrire l'horizon dans lequel les œuvres d'art ont vécu et agi que de savoir comment elles sont nées. « Il ne s'agit pas de présenter les œuvres littéraires dans le contexte de leur temps, mais bien de donner à voir dans le temps où elles sont nées le temps qui les connaît - c'est-àdire le nôtre ${ }^{6}$. Ce serait donc retrancher aux œuvres du passé que d'ignorer leur mode de composition avec le temps, que de laisser pour compte cette trace qu'elles produisent dans l'histoire et qui nous ménage un accès jusqu'à elles. Car ce phénomène de "réfraction » signalé par Benjamin n'affecte pas seulement notre regard mais les œuvres elles-mêmes dans le temps du regard qui se porte sur elles. La nature de ce rapport, toujours susceptible d'être activé et élargi, est telle qu'il échappe au seul principe de réception. Reporter les œuvres à l'horizon de leur action et de leur vie signifie bien plus que donner droit à une postérité qui leur serait attachée : il s'agit de faire apparaître ce qui d'emblée implique l'œuvre dans un devenir, un devenir geste par exemple, ou un devenir politique. Autrement dit, ce devenir des œuvres serait déjà 
inscrit en elles comme une attente, un appel, un ferment, dit W. Benjamin, voire une prophétie, et ceci dans la mesure où « chaque époque possède une possibilité nouvelle, mais non transmissible par héritage, qui lui est propre, d'interpréter les prophéties que l'art des époques antérieures contenait à son adresse. Il n'est pas de tâche plus importante pour l'histoire de l'art que de déchiffrer les prophéties ${ }^{7}$, et, se doit-on d'ajouter, pour l'art lui-même quand la réfraction s'y trouve spécifiquement mise en œuvre, quand le montage qu'il opère n'est pas autre chose que la forme dialectique du déchiffrement : dans La ricotta plus qu'ailleurs, le passé fait du présent qui l'allègue les conditions de son interpolation critique et la peinture fait du cinéma l'espace de transformation de l'avenir qui en elle fermentait.

\section{Interpolation picturale et survivance figurale}

Pasolini s'est exprimé à plusieurs reprises sur les origines picturales de son cinéma. La ricotta met en scène et en ménage deux amours pasoliniens : Masaccio " pour la sobriété du paysage, la simplicité des volumes, la rusticité des illuminations [...]. En revanche, lorsque j'ironise sur la passion bouffonne du réalisateur (Orson Welles) et me caricature moi-même, j'ai en tête les maniéristes, mon autre grande passion: Pontormo, Rosso Fiorentino, les maniéristes florentins $»^{8}$. La ricotta prendrait en charge une dialectique des styles picturaux et des conceptions figurales, faisant doublement jouer la peinture comme « fondement phénoménologique d'une réalité plastique et comme composante d'une unité stylistique $»^{9}$. D'une part, la manière transposée en tableaux vivants, d'autre part le vitalisme mimétique du Quattrocento incarné en la personne de Stracci.

Ce matérialisme vital, cette gravité existentielle de Masaccio, exaltés par Roberto Longhi dans ses leçons - en particulier dans ses notes sur la chapelle Brancacci del Carmine à Florence ${ }^{10}$ - furent pour Pasolini, on le sait, une expérience intellectuelle et esthétique décisive. Sous cet angle, Stracci est bien dépositaire de l'aumento di vitalità perçu par Longhi, ce mouvement qu'il sent venir de l'Antiquité et transiter par Masaccio. La persona de Stracci est bien d'abord un corps intensément et figuralement hérité de Masaccio. Cette empreinte figurale est irréductible à la citation parce qu'irréductible à la signification; elle est affaire d'aspect, de volumétrie, de densité, de frontalité, de gravité. Elle serait de l'ordre de l'obtus et non de l'obvie, pour reprendre la distinction jadis introduite par Roland Barthes ${ }^{11}$. Que Stracci ait à voir avec les figures de Masaccio signifie moins qu'il leur ressemble qu'il ne leur prête corps et mouvement, qu'il n'en figure le type selon le sens que Sergueï Eisenstein donnait à ce mot, lorsqu'il opposait la "figure typique » à la surface trop malléable et dénuée de résistance organique du visage de l'acteur professionnel. Tout au contraire du rapport désinvesti, vide, pour ainsi dire postiche que le Christ-figurant entretient avec son référent pictural, le Christ du tableau de Rosso, le type figural de Masaccio est à l'état de force obtuse dans chaque mouvement de Stracci ou, pour le dire autrement, la figuralité de Masaccio trouve en Stracci son type. Précisons: les personnages des tableaux vivants s'épuisent dans leur fonction, le corps employé se résout et se consomme entièrement dans l'imitation de la figure du tableau tandis que Stracci excède le rôle qu'il doit tenir et le sens même qu'il remplit dans l'économie picturale de la représentation cinématographique; il résiste parce qu'il est non seulement physionomiquement, mimétiquement, mais encore obtusément figure de la peinture de Masaccio. À la signification du corps mimétique des tableaux s'oppose le corps retrouvé 
de Stracci. Il n'y a plus de corps, se désolait Pasolini, ou plutôt n'y aurait-il jamais de corps que de comparution politique d'un corps, et de comparution que frappée d'anachronisme. Stracci, longtemps relégué aux abords de l'histoire et aux marges de la représentation picturale, est un corps retrouvé précisément parce que le lieu de son destin politique est aussi celui de son anachronisme esthétique. Cette dynamique d'intégration par intensification et non par citation amène à repérer une tension à l'œuvre dans $L a$ ricotta entre régime pictural d'interpolation et régime figural de survivance. Approfondir la question de l'interpolation suppose de mettre en évidence le double registre d'intertextualité iconique entre peinture et cinéma - il faudrait alors parler d'une modalité directe et d'une modalité libre de l'interpolation ${ }^{12}$ - mis en place à partir de la fiction du film dans le film. Donner droit à la survivance ouvre en direction d'une anthropologie de la figure ${ }^{13}$. Cependant, interpolation et survivance trouvent dans la forme du tableau vivant une énergie commune.

Le tableau vivant est le moyen scénographique par lequel le cinéma tente d'atteindre en la mimant non pas tant la fixité de l'image de peinture que l'instance suspensive du tableau, ce que l'on pourrait nommer son épochè.

Entrons dans la chapelle Capponi à Santa Felicita à Florence. Qu'avons-nous sous les yeux? Une déposition, un Christ déposé, une déploration, une mise au tombeau, une Pietà, une Pietà disjointe, un adieu fait à Marie, un transport de Christ, une Trinité inchoative, un passage de trône à trône, une extase profane ${ }^{14}$ ? Après tout, comment intituler ce que le retable peint par Pontormo donne à voir? Quelle iconographie rassurante, en effet, si ce tableau met justement en "instance " son sujet, selon l'heureuse formule de Jean-Claude Lebensztejn, si d'une mise au tombeau il fait une mise en instance du tombeau $?^{15}$ Cette suspension regarde à la fois la configuration passionnelle dans laquelle les figures du retable trouvent place et le dispositif de son intégration au sein de la chapelle ${ }^{16}$. La mise en instance a pour effet de déjouer le tableau comme lieu de détermination de l'action, autrement dit de révoquer le rapport de conformité des figures à la signification, et partant de déjouer le tableau comme moment de l'histoire, s'agissant de représenter le drame à partir duquel se réordonnent temps et histoire. Mettre en instance, ce serait cela : temporiser et précipiter en un seul geste. Le premier temps consisterait à régler le visible sur une fonction d'arrêt et de présentation. La critique a généralement sous-estimé la valeur ostensive du tableau de Pontormo et de l'ensemble de la chapelle, qui appelle chez le dévot ou le spectateur un regard extasié. Car cette ostension en peinture est ostension du corps du Christ; elle s'affirme dans la dimension eucharistique du tableau d'autel. Au moment du sacrement eucharistique le Christ en présence vient coïncider avec le Christ en figure. C'est là une coïncidence essentielle à la tension figurale au sein de la forme picturale aussi bien qu'à sa propagation dans la forme cinématographique. Car si cette charge ostensive relève de la peinture en tant que telle - fixer les figures en l'état d'une comparution immuable, donner à voir cette stupéfaction et y ordonner le regard -, Pontormo l'aura intensifiée jusqu'au seuil d'une dislocation qui trouve dans La ricotta à la fois son point de rupture et sa reprise dramaturgique. Ce serait là l'insistance de l'instance. Dans le retable Capponi, la suspension procède d'un mouvement de désincarnation progressive du Christ. Jean-Claude Lebensztejn a justement remarqué, en confrontant le tableau aux études préparatoires, une évolution qui allège par degré et irréalise les corps. C'est particulièrement vrai pour le Christ dont un premier dessin (fig. 3) le montre rasé, lourd et sans grâce, à l'image d'un véritable corps pesant du poids de sa mort. À ce stade, le corps représenté est encore le corps historique du Christ, mais un corps en 
attente d'être revêtu du Christ. Une seconde esquisse, plus conforme à la version finale, se concentre sur la tête et la main qui la soutient en l'orientant, dans celle-ci l'enjeu est visiblement la recherche de l'état d'apesanteur caractéristique du tableau (fig. 4). Cette désubstanciation doit être mise en perspective d'une étude pour l'élévation des âmes $\mathrm{du}$ Jugement dernier du même Pontormo dans la chapelle de San Lorenzo à Florence (fig. 5), étude qui se concentre sur les modalités posturales du port et du transport du corps ressuscité. En bref, tout se passe comme si la configuration figurale et gestuelle de l'instance Capponi accueillait et signifiait le corps du Christ comme un corps déposé dansle même temps qu'élevé, déposé et ressuscité. Cette ambivalence constituée par l'affirmation de la présence du corps historique du Christ en représentation et de la révocation simultanée de sa corporéité terrestre prend tout son sens si on la réfère à la manifestation sacramentelle de la présence réelle du Christ, notamment réaffirmée par Tommaso de Cajetan dans le contexte de controverses des premières décennies du XVIème siècle : «Personne n'est assez fou pour dire que l'esprit est le corps; mais nous disons que le corps du Christ a un mode d'être spirituel dans le sacrement. Il est même présent comme un vrai corps dans le sacrement mais il n'existe pas sous la modalité du corps $»^{17}$.

Fig. 3

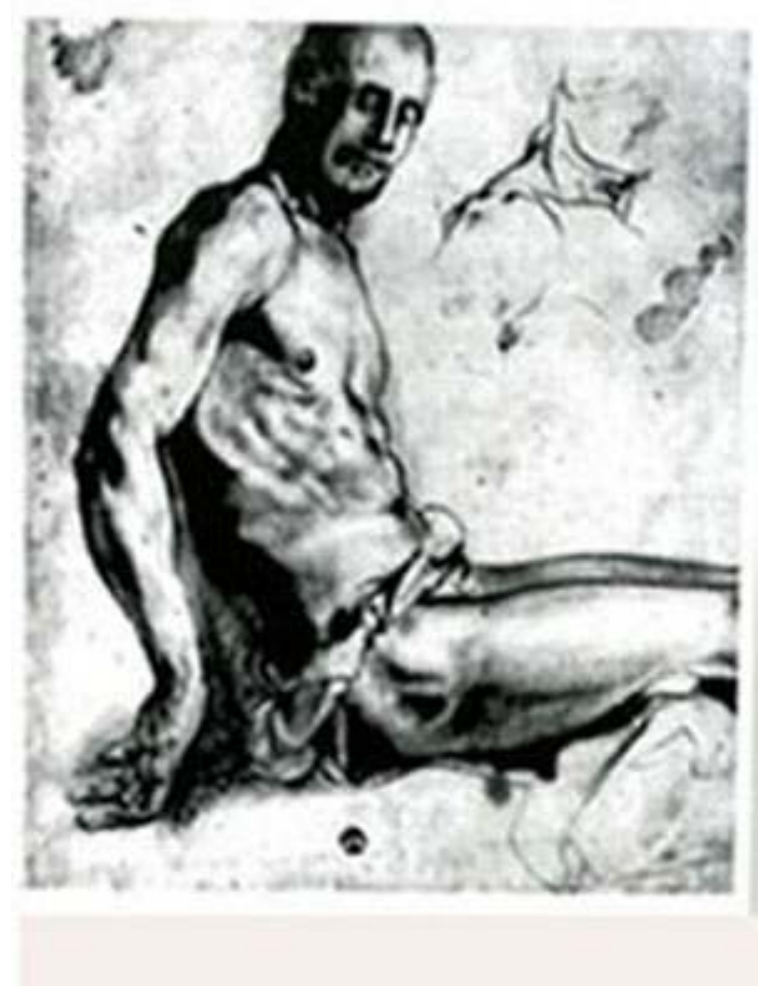

Pontormo, Etude pour le Christ de la Déposition, 1526-27, sanguine, (353 × 280), Galleria degli Uffizi, Florence. 
Fig. 4

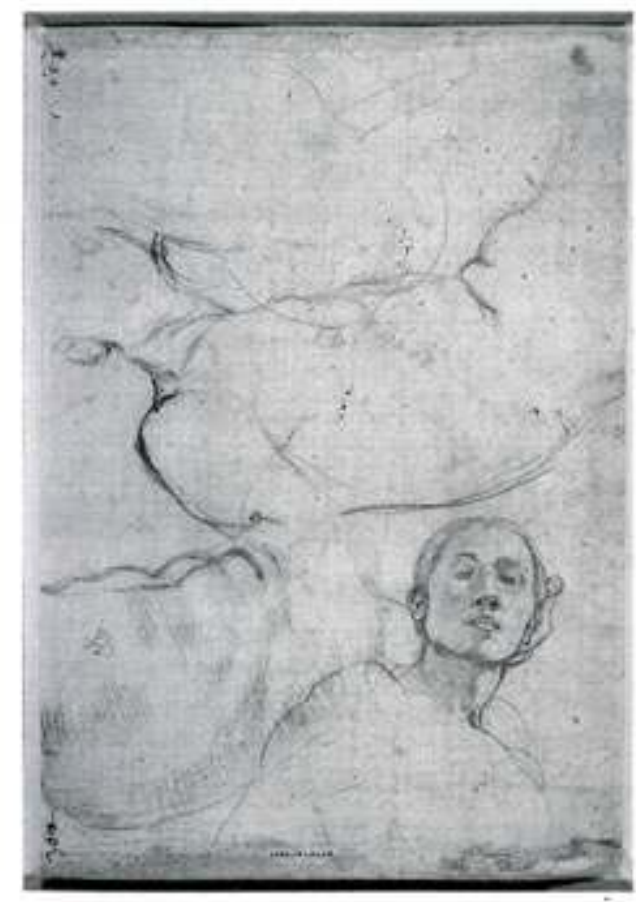

Pontormo, Etude pour le Christ de la Déposition, 1527, sanguine, (142 × 201), London, British Museum.

Fig. 5

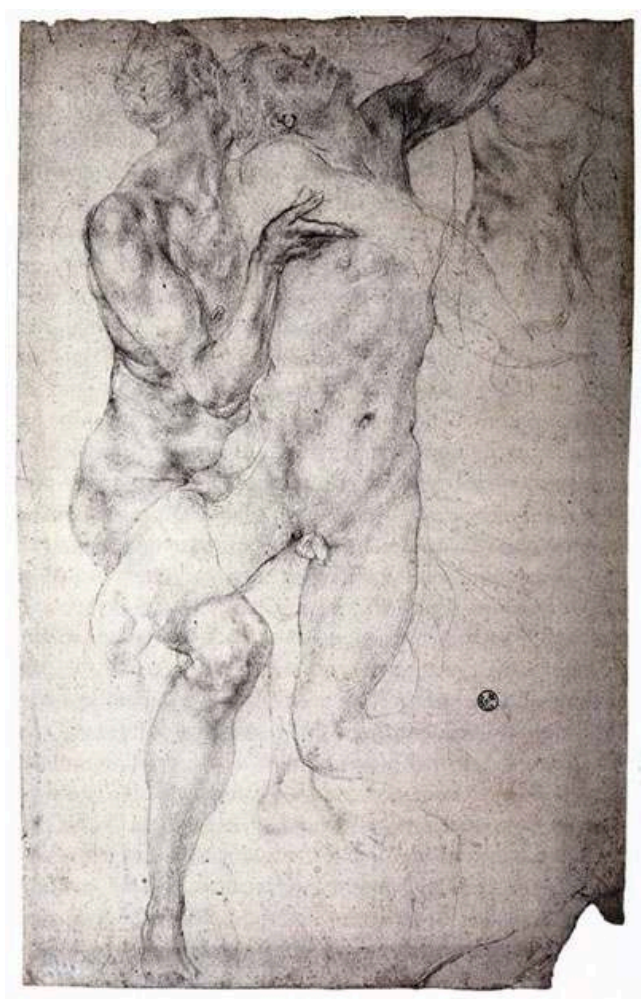

Pontormo, Nu assis qui soutient un nu débout (Etude pour l'ascension des âmes pour Eglise de San Lorenzo, Florence), crayon noir sur papier blanc, Galleria degli Uffizi, Florence. 
13 Le deuxième temps de la mise en instance relève de la fonction d'appel ou d'irrésolution qui fait le pendant du tableau ou du tableau fait un pendant: tout est encore en instance, imminent mais sans cesse différé parce que sur le point d'être délivré, de se déterminer ; ce n'est pas tant que cet instant aurait lieu dans La ricotta, ce qui toutefois advient et l'on verra de quelle façon, c'est surtout que Pasolini fait de cette différance l'espace de détermination anachronique et politique de la figure. Insistance et imminence sont donc les deux temporalités qui se conjoignent dans l'épochè de la représentation Capponi.

14 Une expérience, une constatation que chacun peut faire: ce que la peinture de Pontormo expose est bien toujours en instance devant nos yeux tandis que vient s'interpoler une autre image ou un ensemble d'images en mouvement dont Pasolini est l'auteur. Cependant, le sentiment d'irrémédiable qui colore l'expérience herméneutique en question ne tient pas au simple fait que dans La ricotta Pasolini cite le tableau de Pontormo et donne à cette citation la forme paradoxale d'un tableau vivant. $\mathrm{Au}$ sein du rapport citationnel, un événement se produit qui décroche ou rompt l'instance du retable Capponi et, disons, semble démettre toute instance. Cet événement, par quoi le réel surgit dans l'image ou plus exactement entre les images, c'est une chute : le rappel involontaire et soudain de la matérialité d'un corps - la manifestation de sa gravité, de son pesant. Le Christ en équilibre précaire sur les épaules de porteurs dont les référents picturaux au caractère angélique ne semblent qu'effleurer le sol s'écroule sous les rires.

15 La chute en soi - a fortiori dans le contexte de la mise en scène de la Passion du Christ -, c'est le corps rendu à son essence profane, c'est-à-dire du point de vue dramaturgique, l'expression de la réintégration profane du corps. Cette réintégration est ici l'opération fondamentale par laquelle le cinéma prend en charge la peinture et la peinture livre son instance au cinéma, fondamentale pour Pasolini parce qu'elle ramène au point de partage du sensible, au point de sa relève théologique ou politique, fondamentale encore parce que son effet comique introduit un élément de distanciation et amorce un nouveau processus. Cette chute, cette crise, au sens diagnostique du mot, forme comme une origine au sein du film, non pas l'instant crucial - qui reste la mort de Stracci -, mais l'événement à partir duquel l'intégration du passé s'engage sous le signe de la contamination.

16 Que se passe-t-il en effet ? Du point de vue du schématisme mis en avant par Warburg, la représentation se vide brusquement de son pathos, ou plutôt l'énergie pathétique contenue dans la formule gestuelle du transport du Christ s'échappe du cadre de sa reconstitution, tandis que le sujet du tableau est définitivement imprégné par la confusion et l'insouciance qui règne sur le plateau de tournage. Cette contamination du sacré par le profane est très exactement une profanation au sens d'une désacralisation, d'une dé-séparation (de sacer: ce qui est séparé, mis à part) ${ }^{18}$, mais aussi et simultanément une mobilisation du profane par le sacré. Du drame de la mort du Christ, il ne reste que « le négatif, dit Montani, la trace vide, [c'est-à-dire] le schéma pathétique privé de substance ${ }^{19}$. Ce schéma devenu sans objet, ce schéma purement virtuel n'a rien perdu de sa «force du passé ». Au contraire, on dirait plutôt qu'il la communique au film comme une sorte de "libre énergie», une énergie qui se met à la recherche d'une nouvelle incarnation. « Et bien, c'est dans cette condition que La ricotta fait surgir la figure de Stracci [...] à la place laissée vacante par l'image du Christ après l'écroulement de la composition picturale. ${ }^{20}$ En ce sens, la chute ne témoigne 
d'aucune faillite de l'image à prendre en charge le passé, comme la critique l'a généralement souligné, mais inaugure une nouvelle possibilité. L'instance picturale cède : acte d'ouverture.

Or, il faut voir que cette chute était déjà en peinture - et précisément dans la Descente de croix de Rosso substituée au Couronnement du Christ entre l'écriture du scénario et le tournage $^{21}$-, mais à l'état de menace, là encore en instance de se produire. Dans la Déposition de Volterra peinte par Rosso, «le moment choisi est physiquement précaire mais dramatiquement stable et iconographiquement établi », comme l'a justement vu Lebensztejn ${ }^{22}$. L'imposante croix latine organise la composition dans son format vertical et l'articule en deux parties égales, basse et haute. La césure se situe au niveau des pieds du Christ. Cette division spatiale et compositionnelle nette correspond à un découplage des régimes pathétiques haut et bas. Le groupe de la partie inférieure reconduit la gestualité du compianto selon des formules pathétiques et un agencement rhétorique éprouvés ; équilibre et pondération des postures assurent en accord avec le principe de convenance la répartition de l'affect douloureux sous trois modalités expressives concordantes, tandis que le groupe de la partie supérieure est animé par une dynamique arythmique qui semble désarticuler les corps eux-mêmes. Deux temporalités gestuelles s'exercent simultanément et contrastent l'une, prise en charge par le style aulique qui en exhausse la gravité muette, avec l'autre, incidente et disruptive, au service d'une spectacularité grotesque. Sous cet angle, la perversion du mouvement qui englobe la figure du Christ, quelle qu'en soit la nature, introduit un élément d'indignité comique en soi, en l'espèce d'une gesticulatio. Le tableau repose sur une tension entre la valeur sublime et intemporelle de l'événement (le sacrifice) et la menace prosaïque d'un accident : tout se noue autour de l'instabilité du corps mort du Christ et de l'instance profane de sa chute. Pasolini, on le sait, attribuait au maniérisme la dissolution de toute vision intégrale et harmonieuse du monde: "Le maniérisme était mécréant (miscredente) : Pontormo, Rosso Fiorentino [...] étaient profondément diaboliques et mécréants (erano diabolici, erano miscredenti). ${ }^{23}$ L'épithète "diabolique " appliquée aux figures de Rosso provient de Vasari, elle est reprise par Longhi comme composante de l'art de Rosso dans sa recension de la monographie de P. Barocchi, ainsi que par Briganti dans La Maniera italiana, qui servit à Pasolini d'ouvrage de référence pour la construction des tableaux vivants. Il est de même question de la veine profane sinon « hérétique » de la douleur des figures pontormiennes. Lors du procès qui lui fut intenté pour "outrage à la religion", Pasolini évoque à sa décharge le Christ " profanamente raffigurato " du retable Capponi. Lieu commun en somme que cette crise du sentiment religieux dans la peinture et l'expression maniéristes ${ }^{24}$. Or il me semble que la dystonie et la désynchronisation grotesques de la partie supérieure du tableau de Rosso, dans le soupçon d'impiété qu'elle soulève, visent en réalité le contraste ou l'écart maximal avec le pathétisme théâtral de la moitié inférieure. Les deux sont violemment juxtaposées, selon une asyndète visuelle. Charge comique et charge pathétique sont ainsi aboutées pour produire un choc et un manque visuel, moyen, me semble-t-il, de faire advenir et de relancer par contraste simultané le drame infigurable de la mort du Christ. Le moment choisi dans la séquence narrative qui va du déclouement de la croix jusqu'à la mise au tombeau est celui de plus grande tension entre la culmination de la douleur et la matérialité du corps. C'est sans doute cette tension que Pasolini retient lorsqu'il détourne le pathos de la représentation dans le cinéma en écroulant la composition du retable Capponi, cette force de contraste ou d'incongruence qu'il vise au détriment de la structure mais au bénéfice d'un accroissement d'intensité. En 
montant les deux retables ensemble à l'intérieur des tableaux vivants, Pasolini aura déplacé ou reporté l'instabilité physique au sein de l'iconographie établie de la déposition de Rosso vers l'instabilité dramatique et iconographique du tableau de Pontormo, le burlesque gestuel des figures de Rosso vers la suspension quasi extatique du groupe de Pontormo. En ce sens le cinéaste se sert de Rosso d'un point de vue dramaturgique, il procède au cinéma avec les moyens de Rosso pour dégager le pathos contenu dans la Passion et le libérer, c'est-à-dire le délier de la représentation et le rendre à la possibilité immédiate. La séquence dynamique des tableaux vivants est donc la suivante : déplacement, condensation et contamination.

\section{L'image dialectique du figurant}

En aucun cas la scène du repas de ricotta, ou Stracci show ainsi que l'intitulent les spectateurs internes, n'est désignée comme tableau vivant; filmée en noir et blanc, dans une alternance de champs et contre-champs, en accéléré pour les plans réservés à Stracci, elle ne rentre pas, à première vue, dans l'histoire de la Passion pas plus qu'elle ne s'inscrit dans le plan topographique du plateau de tournage. Cet espace vague de lisière, entre la ville dont on aperçoit au loin le rempart architectural et la campagne romaine clairsemée de ruines fixe le lieu choisi par le réalisateur pour situer le Golgotha infiguré chez Pontormo. Pasolini joue pleinement de cette qualité d'indétermination, en la réalisant topographiquement et en l'actualisant socialement. Avant que de ménager un accès souterrain à ce que l'on peut bien nommer le lieu de Stracci, l'espace est travaillé par la confusion ou l'indistinction entre sacré et profane, pathétique et comique, indistinction qui fait fond à l'opposition sociale entre Stracci et les notables, Stracci et la diva - voire le chien de la diva - entre la famille du cinéma et la famille de Stracci. Il y aurait de Pontormo à Pasolini comme une explication, un dépli du lieu pictural de la mort du Christ dans le lieu du set, comme une actualisation surdéterminée - fût-ce dans l'indétermination - de la pure potentialité ou virtualité topographique du retable Capponi. Au titre du vague, il s'agit de passer du lieu en puissance ou lieu non actualisé de Pontormo au non-lieu actualisé de Pasolini (périphérie de Rome) où vit et surtout meurt Stracci. Dans cette perspective, la topographie figurale de la caverne est également surdéterminée : à la fois tombeau du Christ et lieu par définition grotesque. Le repas de ricotta qui y prend place est dès lors un tableau vivant pour le film que nous voyons, un tableau trop vivant. Sans le savoir, Stracci est en train de réactiver une « scène de genre » dont le titre légende aussi bien le repas auquel nous assistons qu'il intitule le sketch dans son ensemble: Il Mangiaricotta de Vincenzo Campi (fig. 6) ${ }^{25}$. Stracci ne rejoue pas ce tableau à la manière dont Orson Welles fait jouer à ses figurants les scènes de la déposition, il en actualise le contenu grotesque, populaire et comique plutôt qu'il n'en imite la forme. À ce point, et quels que furent les intentions de Pasolini, il est permis de voir dans La Ricotta la reprise et le développement du tableau de Campi sous forme d'une question : n'y a-t-il, semble nous dire Stracci, n'y a-t-il dans le Mangeur de ricotta qu'une figure de la vie basse et matérielle, de l'aliénation grotesque? 
Fig. 6

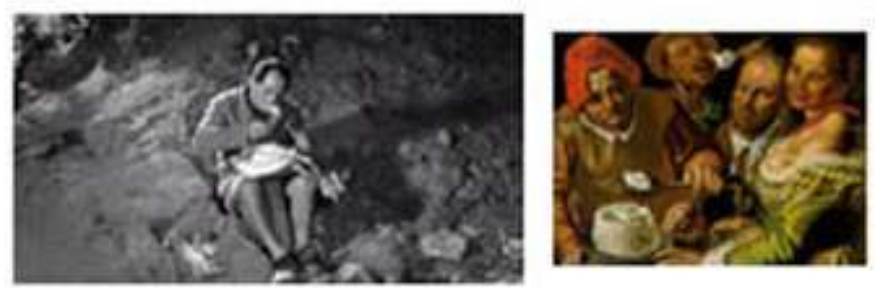

Vincenzo Campi, Mangiaricotta, huile sur toile, $(77 \times 89 \mathrm{~cm})$, Musée des Beaux Arts, Lyon. s'entremêlent théologie et anthropologie chrétiennes. Erich Auerbach, dont Pasolini était un lecteur attentif, a montré que la séparation hiérarchisée des styles comme des contenus dans la tradition gréco-latine (du style élevé au style bas) ne pouvait être maintenue dans la perspective chrétienne puisque, à sa source même, le message chrétien transgresse cette hiérarchie : le Christ s'est abaissé et humilié dans l'homme jusqu'à recevoir une mort d'esclave. "Que le Roi des rois eût été traité comme un vulgaire criminel, qu'il fût moqué, couvert de crachats, battu de verges et finalement cloué sur une croix, anéantit, sitôt qu'il s'imposa à la conscience des hommes, l'esthétique de la séparation des styles. [...] Un nouveau style élevé [se fonde, qui] assume la réalité concrète avec sa laideur, son indignité et sa bassesse $»^{26}$. Or Pasolini intègre justement à l'actualisation grotesque du tableau de Campi un élément pathétique spécifique, celui de la dérision, des rires et des moqueries que subit Stracci. La dérision en tant que telle fait bien partie de l'histoire de la Passion du Christ, elle constitue même le moment exemplaire de l'indéfectible compromission du haut et du bas relevée par Auerbach. On peut alors supposer qu'en dehors du film en train d'être tourné et des tableaux vivants de la Passion, la dérision de Stracci en mangeur de ricotta réactualise aussi l'événement de la dérision du Christ. Cela conduirait à faire de Stracci une lecture dialectique, à voir la persona du figurant Stracci comme une figure et une contre-figure du Christ: sa mort le rend semblable au Christ, d'une conformité qui est aussi l'extrême dérision de la mort du Christ et l'exact renversement du sens de sa Passion. Ce sens pris à rebours n'est pas seulement celui de l'opposition entre le Royaume du ciel promis à l'horizon eschatologique chrétien et le royaume de la satisfaction des besoins terrestres revendiqué par Stracci, il est aussi et surtout celui de la temporalité de l'histoire chrétienne qui voit dans la mort une des conditions de l'assomption et non sa conséquence.

À l'élément du besoin en tant que tel qui fait la dimension épico-religieuse sinon sacrée du sous-prolétaire selon Pasolini ${ }^{27}$ s'ajoute et même se confond un autre élément qui est celui de la passivité, de l'acceptation devant la condition sous-prolétarienne, élément moral qui s'oppose à la révolte, à la conscience historique de classes et à l'engagement révolutionnaire. À tel moment de La ricotta, le figurant qui tient la place du Christ pose une question toute brechtienne à Stracci : «Pourquoi restes-tu auprès de ceux qui t'affament? »Ce serait en effet dans l'usage du pathos qui fait la dramaturgie du film le moment où Pasolini expose au spectateur la raison distanciée qui précise la faute (au sens brechtien) de Stracci. Mais Stracci, outre son fatalisme ou son « innocence » - mot qui définit chez Pasolini l'absence de rapport critique au passé -, pointe alors, dans le cadre d'une société scindée et développée par le néo-capitalisme, la raison utopique contreposée à la Passion, mais qu'il vit, dit-il, à la manière d'une « vocation ». C'est sur 
cette ambiguïté entre action et Passion, cette dialectique entre l'éthos sous-prolétarien et le pathos chrétien immobilisée en une image - Stracci mort sur la croix - que se clôt La ricotta. "Pauvre Stracci! Crever est le seul moyen qu'il ait trouvé pour faire la révolution »: cette dernière réplique prononcée par Orson Welles sonnait comme le testament politique de Stracci, lors du procès elle fut révisée en une formule qui substitue aux moyens révolutionnaires l'expression de la vie en tant que telle, à moins qu'elle ne les confonde ${ }^{28}$.

La ricotta serait donc la prise de conscience, non pas tant de « l'impossibilité de Stracci à être représenté, sinon mort sur la croix $»^{29}$ que celle de donner figure au sousprolétariat, de relever en figure le réprouvé social, en bref de figurer par des moyens neufs - il faut entendre modernes - ceux qui par destin échappent à la visibilité ${ }^{30}$. La lecture marxiste de l'histoire dont se réclame Pasolini ne peut produire seule un modèle figural de contamination des valeurs, il lui faut revenir à des corrélations passées et s'inscrire dans le mouvement qui entremêle théologie et anthropologie chrétiennes. La vision politique de Pasolini est en même temps saisissement d'une énergie (théologie) et d'une mémoire qui a l'image pour instance (peinture). Sur le plan de la représentation entendue comme espace d'avènement des figures, la mimésis cinématographique de la contamination se constitue en champ de force temporel, en articulant dialectiquement deux éléments fondamentaux, d'une part le passé figural du cinéma et d'autre part la figure cinématographique moderne du figurant. Une nouvelle figure naît de ce rapport, de cette constellation dirait Benjamin, figure de contamination qu'incarne Stracci et qu'il décline dans la conjonction de sa présence au présent et de l'intégration de son passé. Ce à quoi nous avons finalement à faire est la construction pathétique et politique du figurant.

\section{NOTES}

1. Jacopo da Pontormo, Déposition de Croix ou Mise au tombeau, 1525-26, huile sur bois, $313 \mathrm{x}$ $192 \mathrm{~cm}$; Rosso Fiorentino, Déposition, 1521, huile sur bois, 375 x $196 \mathrm{~cm}$, Pinacoteca communale, Volterra.

2. Pier Paolo Pasolini, «Il sentimento della storia ", in Saggi sulla letteratura e sull'arte, ed. par Walter Siti \& Silvia De Laude, Milan, Mondadori, 1999, vol. II, p. 2818-2820.

3. Dans Pasolini (op. cit.), on trouve cette "déclaration" de Pasolini (lettre ouverte à Carlo Lizzani à propos de Médée, 1970) : «Per sua natura il cinema non puo' rappresentare il passato. Il cinema rappresenta la realtà attraverso la realtà, un uomo attraverso un uomo. In fatti il passato diviene una metafora del presente : in un rapporto complesso, perché il presente è l'integrazione figurale del passato. »

4. Ailleurs, Pasolini formule ce qu'est une citation au cinéma en des termes qui laissent supposer un mouvement, une opérativité et une efficace : il s'agit de prendre des passages, des moments du style de quelqu'un d'autre pour les "faire en un certain sens revivre en les citant », P.P. Pasolini, Per il cinema, ed. par W. Siti \& F. Zabagli, avec deux écrits de Bernardo Bertolucci et Mario Martone, Milan, Mondadori, 2001, p. 2900. 
5. «La "vita posthuma" della pittura nel cinema ", in Cinema / pittura: dinamiche di scambio, ed. par Leonardo De Franceschi, Torino, Lindau, 2003, p. 32-42, p. 33 (ma traduction). Le présent texte doit beaucoup au travail de Pietro Montani, qui est né en réponse à sa contribution à la conférence " Trasfigurazioni : la pittura nel cinema », qui s'est tenue à Rome du 5 au 11 décembre 2001 dont « La "vita posthuma"... » est la version écrite.

6. Histoire littéraire et science de la littérature, in Oeuvres, trad. M. de Gandillac, R. Rochlitz \& P. Rusch, Gallimard, Paris, 2000, vol. II, p. 274-283, p. 283.

7. Walter Benjamin, Gesammelte Schriften, ed. Theodor W. Adorno Gershom Sholem, nouv. ed. par Rolf Tiedemann \& Herman Schweppenhäuser, Frankfurt am Mein Suhrkamp 1991, vol. I, 3, p. 1046. Ce trait, constitutif de l'œuvre, Gérard Wajcman proposait récemment de lui donner le nom de « décomplétude» : «...Les grandes œuvres ne sont jamais achevées. Non parce qu'elles seraient non finies, mais parce que, même finies, elles s'emplissent des temps qu'elles traversent et se complètent à l'infini des interprétations toujours recommencées qu'elles suscitent ou engendrent; même finies, les œuvres sont manquantes, non pas incomplètes mais plutôt "décomplètes", d'une "décomplétude" essentielle et irrémédiable... », G. Wajcman, « Le regard de l'ange ", in Y voir mieux, y regarder de plus près : autour de Hubert Damisch, ed. par Danièle Cohn, Paris, ENS, « Aesthetica »), 2003, p. 183-198, p. 188.

8. «Masaccio per la sobrietà del paesaggio, la semplicità dei volumi, la rusticità delle illuminazioni [...] Invece nei i passi in cui faccio ironia sul regista (Orson Welles) di questa Passione buffonesca, e metto in caricatura me stesso, ho in mente i manieristi, un altro mio grande amore : Pontormo, Rosso Fiorentino, i manieristi fiorentini », cité par Franca Angelini, Pasolini e lo spettacolo, Roma, Bulzoni, 2000, p. 76.

9. Luc Vancheri, Cinéma et peinture, Paris, Armand Collin, 2007, p. 152.

10. Roberto Longhi, "Gli affreschi del Carmine, Masaccio e Dante», in Edizione delle opere complete, 8.1 : Fatti di Masolino e di Masaccio e altri studi sul Quattrocento : 1910-1967, Firenze,Sansoni, 1975 , p. $67-70$

11. Cf. en particulier R. Barthes « Le troisième sens ", in Cahiers du Cinéma, $n^{\circ}$ 222, 1970, p. 12-19.

12. Dessiner une typologie de la citation s'avère nécessaire mais non suffisant. Les intérêts différés de la peinture dans La Ricotta ne s'épuisent certes pas à dresser le catalogue, en plus des tableaux vivants, des citations indirectes, quand ces intérêts relèvent plus spécifiquement d'un processus de dispersion, ou mieux de dissémination, et d'une pratique du montage.

13. Selon une perspective épistémologique et théorique dont Georges Didi-Huberman trace les linéaments dans Devant le temps : histoire de l'art et anachronisme des images, Paris, Éd. de Minuit, 2000 et L'Image survivante: histoire de l'art et temps des fantômes selon Aby Warburg, Paris, Éd. de Minuit, 2002.

14. Autant de manières d'indexer le sujet de la représentation, de G. Vasari à J.-C. Lebensztejn, en passant par J. Cox Rearick, J. Shearman et L. Steinberg.

15. «C'est Pontormo, qui vers 1524-25, à la chartreuse, avait introduit en toscane, peut-être en Italie, la déposition en instance. La descente de croix projetée pour la chartreuse reprenait la composition de la petite Passion de Dürer, mais Pontormo, en installant le porteur à terre, déplaçait le moment, en faisant une étape transitionnelle, dramatiquement instable. La pietà du même cycle, reprenant elle aussi un bois gravé de Dürer, figure le début de l'acte suivant, le moment où l'on dépose le corps sur la pierre, et qui précède la piétà, précisant ainsi l'instance. C'est l'instance du deuil. Rosso a repris cette idée à Sansepolcro, au moment où Pontormo achevait dans le secret de la chapelle Capponi une nouvelle instance, déchirante et suspendue ", Jean-Claude Lebensztejn, «Fragments de miroir », in Le Journal de Jacopo da Pontormo, trad. par Jean-Claude Lebensztejn avec la collab. d'Alessandro Parronchi, Paris, Aldines, 1992, p. 279.

16. Pour une description et une analyse de la chapelle dans son ensemble, on se reportera également à J.-C. Lebensztejn qui renvoie dos-à-dos les tentatives de J. Shearman et de L. Steinberg dans la mesure où ils privilégient un certain type de rapport, qui entre le tableau et 
l'autel, qui, entre le tableau et la fresque de la coupole, au détriment des rapports d'ensemble et de la double dédicace de la chapelle.

17. Tommaso de Cajetan, 1525, 44, traité publié à Venise en 1531 sous le titre Instructio nuntii circa errores libelli de cena domini, cité par Regina Stefaniak dans un essai très éclairant: "Replicating Mysteries of the Passion: Rosso's Dead Christ with Angels», Renaissance Quartely, vol. 45, n 4, 1992, p. 677-738. p. 682 (ma traduction).

18. Cf. Giorgio Agamben, Profanations, trad. Martin Rueff, Paris, Payot-Rivages, 2006.

19. Pietro Montani, op. cit., p. 41.

20. Ibid., p. 39, 41.

21. Rappelons que le scénario de La Ricotta prévoyait la reconstitution, une reconstitution largement inspirée, de la fresque du «Couronnement » de Pontormo - il s'agit en fait du Christ devant Pilate peint à fresque pour la chartreuse de Galluzzo à Florence. À l'étape de l'écriture, Pasolini condense plusieurs moments de la Passion en un seul, contractant l'histoire de la présentation devant Pilate jusqu'à l'épisode de la dérision et de la molestation: "Le Couronnement de Pontormo, écrit-il. [...] Le Christ est au centre, souffrant, les yeux baissés ; il se laisse mettre la couronne d'épine et subit les moqueries et les coups» (P.P. Pasolini, «La Ricotta ", Sceneggiature e trascrizioni, in Per il Cinema, vol. I, p. 331). Pasolini a finalement substitué La Déposition de Rosso au Couronnement de Pontormo ; celle-la apparaît bien à la place narrative et fonctionnelle dévolue à celui-ci dans le scénario.

22. Tandis que «dans les deux dépositions suivantes, Rosso choisit le moment qui précède la déploration (Sansepolcro, 1527-28) et celui qui la suit à peine (Louvre, après 1530). Ces trois déplorations, étalées sur dix ans ou plus, suivent la chronologie du drame », J.-C. Lebensztejn, op.cit., p. 280.

23. P.P. Pasolini, « L'arte del Romanino », Saggi sulla letteratura e sull'arte, op.cit., vol. II, p. 2794.

24. Harvey E. Hamburgh est l'un des rares historiens à proposer une analyse dévotionnelle de la Déposition de Rosso dans le contexte franciscain qui fut le sien; s'il replace de façon convaincante la figure du Christ dans la perspective eucharistique du tableau d'autel, il ignore néanmoins la répartition dynamique des corps et leur gestualité disruptive, notant simplement que «the full force of meaning in the Volterra Descent comes in the contrast made between the aspect of Christ and the other depicted", H.E. Hamburgh, «Fiorentino's Descent from the Cross in a Franciscan Context », The Sixteenth Century Journal, vol. 19, n 4, 1988, p. 577-604, p. 599.

25. Pour une analyse détaillée de ce tableau, je renvoie à Valérie Boudier, Des comestibles et d'autres sujets du même ordre. Peinture et nourriture dans les scènes de genre de Vincenzo Campi, Bartolomeo Passerotti et Annibale Carracci, Paris, École des hautes études en sciences sociales, Thèse de doctorat, 2007.

26. Erich Auerbach, Mimésis: la représentation de la réalité dans la littérature occidentale, trad. Cornélius Heim, Paris, Gallimard, 1968, p. 83.

27. Cf. P.P. Pasolini, « Una visione del mondo epico-religiosa » (1964, enregistrement d'un débat), in Per il cinema, op. cit. t. II, p. 2844-2879.

28. «Il suo solo modo di fare la rivoluzione » est devenu « Non aveva altro modo di ricordarci che anche lui era vivo ».

29. Selon Franca Angelini, Pasolini e lo spettacolo, Roma, Bulzoni, 2000.

30. Les conditions de possibilité contemporaines de donner figure au pluriel, c'est-à-dire de rendre visage aux invisibles fait l'objet de la réflexion de Georges Didi-Huberman dans «Peuples exposés à disparaître ", Chimères, 2008, n 66-67: Morts ou vifs, extrait d'un travail en cours intitulé Peuples exposés. 
INDEX

Mots-clés : Pasolini (Piere Paolo), manierismo, réfraction, Nachleben, pathos

\section{AUTEUR}

\section{XAVIER VERT}

Historien de l'art, membre du CEHTA (EHESS) et enseignant à l'Ecole Régional des Beaux-Arts de Nantes. Ses recherches portent en particulier sur la Renaissance et la période moderne. Il s'attache à analyser au sein de la tradition figurative occidentale les conditions de possibilité et les modalités de représentation de la figure humaine selon le double mouvement d'une interrogation qui porte sur anthropomorphisme et figurativité, attendu que l'œuvre d'art est le lieu de leur performance. Il s'agit de replacer et de questionner la figure humaine dans le jeu de ses ressemblances et sous ses statuts esthétique, anthropologique, théologique et politique. Il a notamment travaillé sur la figurativité de la damnation dans la fresque du jugement dernier de Michel-Ange, sur l'institution du portrait infamant en Italie du XIII ${ }^{\mathrm{e}}$ au XVI ${ }^{\mathrm{e}}$ siècle, sur les origines du portrait-charge et sur la construction du figurant chez Pasolini. 